\title{
Differential Subordinations Associated with Multiplier Transformations
}

\author{
Adriana Cătaş, Georgia Irina Oros, and Gheorghe Oros \\ Department of Mathematics, Faculty of Science, University of Oradea, 1 University Street,
} 410087 Oradea, Romania

Correspondence should be addressed to Adriana Cătaş, acatas@gmail.com

Received 26 February 2008; Accepted 15 May 2008

Recommended by Ferhan Atici

The authors introduce new classes of analytic functions in the open unit disc which are defined by using multiplier transformations. The properties of these classes will be studied by using techniques involving the Briot-Bouquet differential subordinations. Also an integral transform is established.

Copyright ( 92008 Adriana Cătaş et al. This is an open access article distributed under the Creative Commons Attribution License, which permits unrestricted use, distribution, and reproduction in any medium, provided the original work is properly cited.

\section{Introduction and definitions}

Let $\mathscr{H}$ be the class of analytic functions in the open unit disc

$$
U=\{z \in \mathbb{C}:|z|<1\}
$$

and let $\mathscr{H}[a, n]$ be the subclass of $\mathscr{H}$ consisting of functions of the form $f(z)=a+a_{n} z^{n}+$ $a_{n+1} z^{n+1}+\cdots$. Let $\mathcal{A}(p, n)$ denote the class of functions $f(z)$ normalized by

$$
f(z)=z^{p}+\sum_{k=p+n}^{\infty} a_{k} z^{k} \quad(p, n \in \mathbb{N}:=\{1,2,3, \ldots\})
$$

which are analytic in the open unit disc. In particular, we set

$$
\mathcal{A}(p, 1):=\mathcal{A}_{p}, \quad \mathcal{A}(1, n):=\mathcal{A}(n), \quad \mathcal{A}(1,1):=\mathcal{A}=\mathcal{A}_{1} .
$$

If a function $f(z)$ belongs to the class $\mathcal{A}(n)$, it has the form

$$
f(z)=z+\sum_{k=n+1}^{\infty} a_{k} z^{k} \quad(n \in \mathbb{N}:=\{1,2,3, \ldots\}) .
$$


For two functions $f(z)$ given by (1.4) and for $g(z)$ given by

$$
g(z)=z+\sum_{k=n+1}^{\infty} b_{k} z^{k} \quad(n \in \mathbb{N})
$$

the Hadamard product (or convolution) $(f * g)(z)$ is defined, as usual, by

$$
(f * g)(z):=z+\sum_{k=n+1}^{\infty} a_{k} b_{k} z^{k}:=(g * f)(z) .
$$

If $f$ and $g$ are analytic in $U$, we say that $f$ is subordinate to $g$, written symbolically as

$$
f \prec g \text { or } f(z) \prec g(z)(z \in U)
$$

if there exists a Schwarz function $w(z)$ in $U$ which is analytic in $U$ with $w(0)=0$ and $|w(z)|<1$ such that $f(z)=g(w(z)), z \in U$.

We consider the following multiplier transformations.

Definition 1.1 (see [1]). Let $f \in \mathcal{A}(p, n)$. For $\delta, \lambda \in \mathbb{R}, \lambda \geq 0, \delta \geq 0, l \geq 0$, define the multiplier transformations $I_{p}(\delta, \lambda, l)$ on $\mathcal{A}(p, n)$ by the following infinite series:

$$
I_{p}(\delta, \lambda, l) f(z):=z^{p}+\sum_{k=p+n}^{\infty}\left[\frac{p+\lambda(k-p)+l}{p+l}\right]^{\delta} a_{k} z^{k} .
$$

It follows from (1.8) that

$$
\begin{gathered}
I_{p}(0, \lambda, l) f(z)=f(z), \\
(p+l) I_{p}(2, \lambda, l) f(z)=[p(1-\lambda)+l] I_{p}(1, \lambda, l) f(z)+\lambda z\left(I_{p}(1, \lambda, l) f(z)\right)^{\prime}, \\
I_{p}\left(\delta_{1}, \lambda, l\right)\left(I_{p}\left(\delta_{2}, \lambda, l\right) f(z)\right)=I_{p}\left(\delta_{2}, \lambda, l\right)\left(I_{p}\left(\delta_{1}, \lambda, l\right) f(z)\right) .
\end{gathered}
$$

Remark 1.2 (see [1]). For $p=1, l=0, \lambda \geq 0, \delta=m, m \in \mathbb{N}_{0}, \mathbb{N}_{0}=\mathbb{N} \cup\{0\}$, the operator $D_{\lambda}^{m}:=$ $I_{1}(m, \lambda, 0)$ was introduced and studied by Al-Oboudi [2] which is reduced to the Sălăgean differential operator [3] for $\lambda=1$. The operator $I_{l}^{m}:=I_{1}(m, 1, l)$ was studied recently by Cho and Srivastava [4] and by Cho and Kim [5]. The operator $I_{m}:=I_{1}(m, 1,1)$ was studied by Uralegaddi and Somanatha [6], the operator $D_{\lambda}^{\delta}:=I_{1}(\delta, \lambda, 0)$ was introduced by Acu and Owa [7] and the operator $I_{p}(m, l):=I_{p}(m, 1, l)$ was investigated recently by Sivaprasad Kumar et al. [8].

If $f$ is given by (1.2), then we have

$$
I_{p}(\delta, \lambda, l) f(z)=\left(f * \varphi_{p, \lambda, l}^{\delta}\right)(z),
$$

where

$$
\varphi_{p, \lambda, l}^{\delta}(z)=z^{p}+\sum_{k=p+n}^{\infty}\left[\frac{p+\lambda(k-p)+l}{p+l}\right]^{\delta} z^{k} .
$$

In particular, we set

$$
I_{1}(\delta, \lambda, l) f(z):=I(\delta, \lambda, l) f(z) .
$$

In order to prove our main results, we will make use of the following lemmas. 
Lemma 1.3 (see [9]). For real or complex numbers $a, b$, and $c\left(c \notin \mathbb{Z}_{0}^{-}:=\{0,-1,-2, \ldots\}\right)$, the following hold:

$$
\begin{gathered}
\int_{0}^{1} t^{b-1}(1-t)^{c-b-1}(1-t z)^{-a} d t=\frac{\Gamma(b) \Gamma(c-b)}{\Gamma(c)} \cdot{ }_{2} F_{1}(a, b ; c ; z) \quad(\operatorname{Re} c>\operatorname{Re} b>0), \\
{ }_{2} F_{1}(a, b ; c ; z)=(1-z)^{-a} \cdot{ }_{2} F_{1}\left(a, c-b ; c ; \frac{z}{z-1}\right), \\
{ }_{2} F_{1}(a, b ; c ; z)={ }_{2} F_{1}(b, a ; c ; z), \\
(b+1) \cdot{ }_{2} F_{1}(1, b ; b+1 ; z)=(b+1)+b z \cdot{ }_{2} F_{1}(1, b+1 ; b+2 ; z), \\
{ }_{2} F_{1}\left(a, b ; \frac{a+b+1}{2} ; \frac{1}{2}\right)=\frac{\sqrt{\pi} \cdot \Gamma((a+b+1) / 2)}{\Gamma((a+1) / 2) \Gamma((b+1) / 2)} .
\end{gathered}
$$

Lemma 1.4 (see [10]). Let $\beta, \gamma \in \mathbb{C}, \beta \neq 0$ and let $h$ be convex in $U$, with

$$
\operatorname{Re}[\beta h(z)+\gamma]>0 \quad(z \in U) .
$$

If the function $p \in \mathscr{L}[h(0), n]$, then

$$
p(z)+\frac{z p^{\prime}(z)}{\beta p(z)+\gamma} \prec h(z) \Longrightarrow p(z) \prec h(z) .
$$

Lemma 1.5 (see [11]). Let $\mu$ be a positive measure on the unit interval $I=[0,1]$. Let $g(t, z)$ be a function analytic in $[0,1] \times U$, for each $t \in I$ and integrable in $t$, for each $z \in U$ and for almost all $t \in I$. Suppose also that

$$
\operatorname{Re}\{g(t, z)\}>0 \quad(z \in U ; t \in I)
$$

$g(t,-r)$ is real for real $r$ and

$$
\operatorname{Re} \frac{1}{g(t, z)} \geq \frac{1}{g(t,-r)} \quad(|z| \leq r<1, t \in I)
$$

If

$$
g(z)=\int_{I} g(t, z) d \mu(t)
$$

then

$$
\operatorname{Re}\left(\frac{1}{g(z)}\right) \geq \frac{1}{g(-r)} \quad(|z| \leq r<1)
$$

Lemma 1.6 (see [12]). Let $\psi(z)$ be univalent in the unit disc $U$ and let $v$ and $\varphi$ be analytic in a domain $D \supset \psi(U)$ with $\varphi(w) \neq 0$, when $w \in \psi(U)$. Set

$$
Q(z):=z \psi^{\prime}(z) \varphi(\psi(z)), \quad h(z):=v(\psi(z))+Q(z) .
$$


Suppose that

(1) $Q(z)$ is starlike in $U$ and

(2) $\operatorname{Re}\left(z h^{\prime}(z) / Q(z)\right)>0$ for $z \in U$.

If $q(z)$ is analytic in $U$, with $q(0)=\psi(0), q(U) \subset D$, and

$$
v(q(z))+z q^{\prime}(z) \varphi(q(z)) \prec v(\psi(z))+z \psi^{\prime}(z) \varphi(\psi(z)),
$$

then $q(z) \prec \psi(z)$ and $\psi(z)$ is the best dominant.

Lemma 1.7 (see $[12$, Theorem 3.3d]). Let $\beta, \gamma, A \in \mathbb{C}$, with $\operatorname{Re}[\beta+\gamma]>0$ and let $B \in[-1,0]$ satisfy either

$$
\operatorname{Re}\left[\beta(1+A B)+\gamma\left(1+B^{2}\right)\right] \geq|\beta A+\bar{\beta} B+B(\gamma+\bar{\gamma})|
$$

when $B \in(-1,0]$, or

$$
\beta(1+A)>0, \quad \operatorname{Re}[\beta(1-A)+2 \gamma] \geq 0,
$$

when $B=-1$. If $p \in \mathscr{\ell}[1, n]$ satisfies

$$
p(z)+\frac{z p^{\prime}(z)}{\beta p(z)+\gamma} \prec \frac{1+A z}{1+B z}
$$

then

$$
p(z) \prec q(z)=q_{n}(z) \prec \frac{1+A z}{1+B z}
$$

where $q_{n}$ is the univalent solution of the differential equation

$$
q(z)+\frac{n z q^{\prime}(z)}{\beta q(z)+\gamma}=\frac{1+A z}{1+B z} .
$$

In addition, the function $q_{n}$, is the best $(1, n)$-dominant and the function $q_{n}$ is given by

$$
q(z)=\frac{\beta+\gamma}{\beta}\left[\frac{k(z)}{K(z)}\right]^{\beta / n}-\frac{\gamma}{\beta}=\frac{z K^{\prime}(z)}{K(z)}=\frac{1}{\beta g(z)}-\frac{\gamma}{\beta^{\prime}}
$$

where

$$
\begin{aligned}
k(z) & =z \exp \int_{0}^{z}(h(t)-1) t^{-1} d t \\
K(z) & =\left[\frac{\beta+\gamma}{n z^{\gamma / n}} \int_{0}^{z} k^{\beta / n}(t) t^{(\gamma / n)-1} d t\right]^{n / \beta},
\end{aligned}
$$

and the univalent function $g$ is given by

$$
g(z)=\frac{1}{n} \int_{0}^{1}\left[\frac{k(t z)}{k(z)}\right]^{\beta / n} t^{(\gamma / n)-1} d t
$$

Now we define new classes of analytic functions by using the multiplier transformations $I(m, \lambda, l)$ defined by $(1.8)$ as follows. 


\section{Main results}

Definition 2.1. Let $-1 \leq B<A \leq 1, \lambda>0, l \geq 0, m \in \mathbb{N} \cup\{0\}$. A function $f \in \mathcal{A}(n)$ is said to be in the class $S(m, \lambda, l ; A, B)$ if it satisfies the following subordination:

$$
\frac{l+1}{\lambda} \cdot \frac{I(m+1, \lambda, l) f(z)}{I(m, \lambda, l) f(z)}-\frac{1-\lambda+l}{l+1} \prec \frac{1+A z}{1+B z} \quad(z \in U)
$$

Remark 2.2. We note that

$$
\begin{aligned}
& S(0,1,0 ; 1-2 \alpha,-1) \equiv S^{*}(\alpha), \\
& S(1,1,0 ; 1-2 \alpha,-1) \equiv K(\alpha),
\end{aligned}
$$

where $S^{*}(\alpha)$ and $K(\alpha)(0 \leq \alpha<1)$ denote the subclasses of functions in $\mathcal{A}$ which are, respectively, starlike of order $\alpha$ and convex of order $\alpha$ in $U$. Also we have the class

$$
S(m, \lambda, 0 ; A, B) \equiv S_{\curlywedge}^{m}(A, B)
$$

studied by Patel [13].

Let $\phi(z)$ be analytic in $U$ and $\phi(0)=1$. We introduce the following definition.

Definition 2.3. A function $f \in \mathcal{A}(n)$ is said to be in the class $\mathcal{A}(m, \lambda, l, n ; \phi)$ if it satisfies the following subordination:

$$
\frac{I(m+1, \lambda, l) f(z)}{I(m, \lambda, l) f(z)} \prec \phi(z), \quad(z \in U)
$$

Remark 2.4. We note that the classes $\mathcal{A}(m, 1, l, n ; \phi)$ were investigated recently by Sivaprasad Kumar et al. [8].

Theorem 2.5. Let $-1 \leq B<A \leq 1, l \geq 0, \lambda>0$, and

$$
(1-B)(1-\lambda+l)+\lambda(1-A)>0 .
$$

(i) Then

$$
S(m+1, \lambda, l ; A, B) \subset S(m, \lambda, l ; A, B) .
$$

Further, for $f \in S(m+1, \lambda, l ; A, B)$, the following hold:

$$
\frac{l+1}{\lambda} \cdot \frac{I(m+1, \lambda, l) f(z)}{I(m, \lambda, l) f(z)}-\frac{1-\lambda+l}{l+1} \prec q(z) \prec \frac{1+A z}{1+B z}
$$


where

$$
q(z)= \begin{cases}\frac{1}{(1 / n) \int_{0}^{1} s^{(l+1) / \lambda n-1}((1+B z s) /(1+B z))^{(1 / n)(A / B-1)} d s}-\frac{1-\lambda+l}{\lambda} & \text { if } B \neq 0, \\ \frac{1}{(1 / n) \int_{0}^{1} s^{(l+1) / \lambda n-1} \cdot \exp (A z(s-1) / n) d s}-\frac{1-\lambda+l}{\lambda} & \text { if } B=0,\end{cases}
$$

and $q$ is the best dominant of (2.7).

(ii) Furthermore, in addition to (2.5), one consider the inequality

$$
A \leq-\frac{B[l+1+\lambda(n-1)]}{\lambda},
$$

where $-1 \leq B<0$, then

$$
S(m+1, \lambda, l ; A, B) \subset S(m, \lambda, l ; 1-2 \eta,-1),
$$

where

$$
\eta=\left\{\left[{ }_{2} F_{1}\left(1, \frac{1}{n}\left(1-\frac{A}{B}\right), \frac{l+1}{\lambda n}+1, \frac{B}{B-1}\right)\right]^{-1}-(1-\lambda+l)\right\} / \lambda .
$$

The result is the best possible.

Proof. Setting

$$
x(z):=\frac{z(I(m, \lambda, l) f(z))^{\prime}}{I(m, \lambda, l) f(z)} \quad(z \in U),
$$

we note that $\chi(z)$ is analytic in $U$ and

$$
x(z)=1+a_{n} z^{n}+a_{n+1} z^{n+1}+\cdots
$$

Using the identity

$$
(l+1) I(m+1, \lambda, l) f(z)=(1-\lambda+l) I(m, \lambda, l) f(z)+\lambda z(I(m, \lambda, l) f(z))^{\prime}
$$

in definition of $\chi(z)$ and carrying out logarithmic differentiation in the resulting equation, one obtains

$$
\frac{z(I(m+1, \lambda, l) f(z))^{\prime}}{I(m+1, \lambda, l) f(z)}=\chi(z)+\frac{z \chi^{\prime}(z)}{\chi(z)+(1-\lambda+l) / \lambda} .
$$

Since $f \in S(m+1, \lambda, l ; A, B)$, we get

$$
x(z)+\frac{z \chi^{\prime}(z)}{X(z)+(1-\lambda+l) / \lambda} \prec \frac{1+A z}{1+B z} .
$$


By applying Lemma 1.4, we obtain that

$$
x(z) \prec \frac{1+A z}{1+B z} .
$$

Hence we have shown the inclusion (2.6). Also, making use of Lemma 1.7 with $\beta=1$ and $\gamma=(1-\lambda+l) / \lambda, q$ is the best dominant of (2.7) and $q$ is defined by (2.8). This proves part (i) of Theorem 2.5.

To establish (2.10), we need to show that

$$
\inf _{|z|<1} \operatorname{Re}\{q(z)\}=q(-1) \text {. }
$$

The proof of the assertion (2.18) will be deduced on the same lines as in [14] making use of Lemma 1.5. If we set $a=(1 / n)(1-A / B), b=(l+1) / \lambda n, c=(l+1) / \lambda n+1, B<0$, then by using (1.13), (1.14), and (1.15), we find from (2.8) that

$$
q(z)=\frac{1}{(1 / n) Q(z)}-\frac{1-\lambda+l}{\lambda},
$$

where

$$
\begin{aligned}
Q(z) & =(1+B z)^{a} \int_{0}^{1} s^{b-1}(1+B s z)^{-a} d s \\
& =\frac{\Gamma(b)}{\Gamma(c)} \cdot{ }_{2} F_{1}\left(1, a ; c ; \frac{B z}{B z+1}\right) .
\end{aligned}
$$

By using (1.13), the above equality yields

$$
Q(z)=\int_{0}^{1} g(s, z) d \mu(s)
$$

where

$$
\begin{aligned}
g(s, z) & =\frac{1+B z}{1+(1-s) B z} \\
d \mu(s) & =\frac{\Gamma(c)}{\Gamma(a) \Gamma(c-a)} s^{a-1}(1-s)^{c-a-1} d s
\end{aligned}
$$

is a positive measure on the closed interval $[0,1]$.

For $-1 \leq B<1$, we note that $\operatorname{Re} g(s, z)>0, g(s,-r)$ is real for $0 \leq r<1$ and $s \in[0,1]$ and

$$
\operatorname{Re}\left\{\frac{1}{g(s, z)}\right\} \geq \frac{1-(1-s) B r}{1-B r}=\frac{1}{g(s,-r)}, \quad|z| \leq r<1 .
$$

Therefore, by using Lemma 1.5, one obtains

$$
\operatorname{Re}\left\{\frac{1}{Q(z)}\right\} \geq \frac{1}{Q(-r)}, \quad|z| \leq r<1
$$

which, upon letting $r \rightarrow 1^{-1}$, yields

$$
\operatorname{Re}\left\{\frac{1}{Q(z)}\right\} \geq \frac{1}{Q(-1)} .
$$

Now, the assertion (2.18) follows by using Lemma 1.5. The result is the best possible and $q$ is the best dominant of (2.7). This completes the proof of Theorem 2.5. 
Taking $m=0, n=1, \lambda=1, l=0, A=1-2 \alpha, B=-1$ in Theorem 2.5, we get the following result due to MacGregor [15].

Corollary 2.6. For $0 \leq \alpha<1$, one obtains

$$
K(\alpha) \subset S^{*}\left(\eta_{1}\right),
$$

where

$$
\eta_{1}=\left[{ }_{2} F_{1}\left(1,2(1-\alpha), 2, \frac{1}{2}\right)\right]^{-1}= \begin{cases}\frac{1-2 \alpha}{2^{2(1-\alpha)}\left(1-2^{2 \alpha-1}\right)}, & \alpha \neq \frac{1}{2} \\ \frac{1}{2 \ln 2}, & \alpha=\frac{1}{2} .\end{cases}
$$

The result is the best possible.

Theorem 2.7. Let $\psi(z)$ be univalent in $U$ with $\psi(0)=1, \operatorname{Re} \psi(z)>0$, and let $z \psi^{\prime}(z) / \psi(z)$ be starlike in U. Let $\phi(z)$ be defined by

$$
\phi(z):=\frac{\lambda}{l+1}\left(\frac{l+1}{\lambda} \psi(z)+\frac{z \psi^{\prime}(z)}{\psi(z)}\right) .
$$

Then

$$
\mathcal{A}(m+1, \lambda, l, n ; \phi) \subset \mathcal{A}(m, \lambda, l, n ; \psi) .
$$

Proof. Setting

$$
q(z)=\frac{I(m+1, \lambda, l) f(z)}{I(m, \lambda, l) f(z)}
$$

we note that $q(z)$ is analytic in $U$.

By a simple computation, we observe from (2.30) that

$$
\frac{z q^{\prime}(z)}{q(z)}=\frac{z(I(m+1, \lambda, l) f(z))^{\prime}}{I(m+1, \lambda, l) f(z)}-\frac{z(I(m, \lambda, l) f(z))^{\prime}}{I(m, \lambda, l) f(z)} .
$$

Making use of the identity (2.14), one obtains from (2.31)

$$
\frac{I(m+2, \lambda, l) f(z)}{I(m+1, \lambda, l) f(z)}=\frac{\lambda}{l+1}\left(\frac{l+1}{\lambda} q(z)+\frac{z q^{\prime}(z)}{q(z)}\right) .
$$

By the hypothesis of Theorem 2.7 that $f$ belongs to the class $\mathcal{A}(m+1, \lambda, l, n ; \phi)$ and in view of (2.32), we have

$$
\frac{\lambda}{l+1}\left(\frac{l+1}{\lambda} q(z)+\frac{z q^{\prime}(z)}{q(z)}\right) \prec \frac{\lambda}{l+1}\left(\frac{l+1}{\lambda} \psi(z)+\frac{z \psi^{\prime}(z)}{\psi(z)}\right) .
$$

If we let

$$
Q(z):=z \psi^{\prime}(z) \varphi(\psi(z))
$$


where

$$
\begin{gathered}
\varphi(\psi(z))=\frac{\lambda}{l+1} \cdot \frac{1}{\psi(z)} \\
h(z):=\psi(z)+Q(z)
\end{gathered}
$$

and since $Q(z)$ is starlike, our theorem is an immediate consequence of Lemma 1.6.

Theorem 2.8. Let $\psi$ be univalent in $U, \psi(0)=1$ and let $\gamma$ be a complex number. Suppose that

(1) $\operatorname{Re}[\lambda(\gamma+1)-(l+1)+(l+1) \psi(z)]>0$ and

(2) $z \psi^{\prime}(z) /(\lambda(\gamma+1)-(l+1)+(l+1) \psi(z))$ is starlike in $U$.

Let the function $F(z)$ be defined by

$$
F(z):=\frac{\gamma+1}{z^{\gamma}} \int_{0}^{z} t^{\gamma-1} f(t) d t
$$

and the function

$$
h(z):=\psi(z)+\frac{\lambda z \psi^{\prime}(z)}{\lambda(\gamma+1)-(l+1)+(l+1) \psi(z)}
$$

then $f \in \mathcal{A}(m, \lambda, l, n ; h)$ implies $F \in \mathcal{A}(m, \lambda, l, n ; \psi)$.

Proof. From the definition of $F(z)$ and

$$
(\gamma+1) I(m, \lambda, l) f(z)=\frac{l+1}{\lambda} I(m+1, \lambda, l) F(z)+\left(\gamma-\frac{1-\lambda+l}{\lambda}\right) I(m, \lambda, l) F(z),
$$

if we let

$$
q(z):=\frac{I(m+1, \lambda, l) F(z)}{I(m, \lambda, l) F(z)}
$$

then we note that $q(z)$ is analytic in $U$. Using (2.38) and (2.39), one obtains

$$
(\gamma+1) \frac{I(m, \lambda, l) f(z)}{I(m, \lambda, l) F(z)}=\gamma-\frac{1-\lambda+l}{\ell}+\frac{l+1}{\ell} q(z)
$$

Differentiating this equality, we obtain

$$
\frac{I(m+1, \lambda, l) f(z)}{I(m, \lambda, l) f(z)}=q(z)+\frac{\lambda z q^{\prime}(z)}{\lambda(\gamma+1)-(l+1)+(l+1) q(z)} .
$$

For $f \in \mathcal{A}(m, \lambda, l, n ; h)$, we have from (2.41)

$$
q(z)+\frac{\lambda z q^{\prime}(z)}{\lambda(\gamma+1)-(l+1)+(l+1) q(z)} \prec \psi(z)+\frac{\lambda z \psi^{\prime}(z)}{\lambda(\gamma+1)-(l+1)+(l+1) \psi(z)}
$$


If we let

$$
Q(z):=z \psi^{\prime}(z) \varphi(\psi(z))
$$

where

$$
\begin{gathered}
\varphi(\psi(z))=\frac{\lambda}{\lambda(\gamma+1)-(l+1)+(l+1) \psi(z)}, \\
h(z):=v(\psi(z))+Q(z), \\
v(\psi(z)):=\psi(z)
\end{gathered}
$$

and since $Q(z)$ is starlike in $U$, our theorem is an immediate consequence of Lemma 1.6.

Theorem 2.9. Let $f(z) \in \mathcal{A}(n)$. Then $f$ belongs to the class $\mathcal{A}(m, \lambda, l, n ; \chi)$ if and only if $F(z)$ defined by

$$
F(z):=\frac{l+1}{z^{(1-\lambda+l) / \lambda}} \int_{0}^{z} t^{(1-\lambda+l) / \lambda-1} f(t) d t
$$

belongs to the class $\mathcal{A}(m+1, \lambda, l, n ; \chi)$.

Proof. From the definition of $F(z)$, we have

$$
\frac{1-\lambda+l}{\lambda} F(z)+z F^{\prime}(z)=(l+1) f(z) .
$$

By convoluting (2.46) with the function

$$
u(m, \lambda, l, n ; z):=z+\sum_{k=n+1}^{\infty}\left[\frac{1+\lambda(k-1)+l}{l+1}\right]^{m} z^{k}
$$

and using a convolution property

$$
z(f * g)^{\prime}(z)=f(z) * z g^{\prime}(z),
$$

one obtains

$$
(l+1) I(m, \lambda, l) f(z)=\frac{1-\lambda+l}{\curlywedge} I(m, \lambda, l) F(z)+z(u(m, \lambda, l, n ; z) * F(z))^{\prime},
$$

that is,

$$
(l+1) I(m, \lambda, l) f(z)=\frac{1-\lambda+l}{\lambda} I(m, \lambda, l) F(z)+z(I(m, \lambda, l) F(z))^{\prime} .
$$

By using identity (2.14), we get

$$
I(m, \lambda, l) f(z)=\frac{1}{\lambda} I(m+1, \lambda, l) F(z) .
$$


Also, we obtain

$$
\begin{aligned}
(l+1) I(m+1, \lambda, l) f(z) & =(1-\lambda+l) I(m, \lambda, l) f(z)+\lambda z(I(m, \lambda, l) f(z))^{\prime} \\
& =\frac{1-\lambda+l}{\curlywedge} I(m+1, \lambda, l) F(z)+z(I(m+1, \lambda, l) F(z))^{\prime} \\
& =\frac{l+1}{\lambda} I(m+2, \lambda, l) F(z) .
\end{aligned}
$$

From (2.51) and (2.52), we get

$$
\frac{I(m+2, \lambda, l) F(z)}{I(m+1, \lambda, l) F(z)}=\frac{I(m+1, \lambda, l) f(z)}{I(m, \lambda, l) f(z)} .
$$

By the hypothesis of Theorem 2.9 that

$$
\frac{I(m+1, \lambda, l) f(z)}{I(m, \lambda, l) f(z)} \prec x(z)
$$

and using (2.53), the desired result follows at once.

\section{References}

[1] A. Cătaş, "Sandwich theorems associated with new multiplier transformations," submitted.

[2] F. M. Al-Oboudi, "On univalent functions defined by a generalized Sălăgean operator," International Journal of Mathematics and Mathematical Sciences, vol. 2004, no. 27, pp. 1429-1436, 2004.

[3] G. Ş. Sălăgean, "Subclasses of univalent functions," in Complex Analysis-Fifth Romanian-Finnish Seminar, Part 1, vol. 1013 of Lecture Notes in Mathematics, pp. 362-372, Springer, Berlin, Germany, 1983.

[4] N. E. Cho and H. M. Srivastava, "Argument estimates of certain analytic functions defined by a class of multiplier transformations," Mathematical and Computer Modelling, vol. 37, no. 1-2, pp. 39-49, 2003.

[5] N. E. Cho and T. H. Kim, "Multiplier transformations and strongly close-to-convex functions," Bulletin of the Korean Mathematical Society, vol. 40, no. 3, pp. 399-410, 2003.

[6] B. A. Uralegaddi and C. Somanatha, "Certain classes of univalent functions," in Current Topics in Analytic Function Theory, pp. 371-374, World Scientific, River Edge, NJ, USA, 1992.

[7] M. Acu and S. Owa, "Note on a class of starlike functions," in Proceeding of the International Short Joint Work on Study on Calculus Operators in Univalent Function Theory, pp. 1-10, RIMS, Kyoto, Japan, August 2006.

[8] S. Sivaprasad Kumar, H. C. Taneja, and V. Ravichandran, "Classes of multivalent functions defined by Dziok-Srivastava linear operator and multiplier transformation," Kyungpook Mathematical Journal, vol. 46, no. 1, pp. 97-109, 2006.

[9] E. T. Whittaker and G. N. Watson, A Course of Modern Analysis: An Introduction to the General Theory of Infinite Processes and of Analytic Functions; with an Account of the Principal Transcendental Functions, Cambridge University Press, Cambridge, UK, 4 edition, 1927.

[10] P. Eenigenburg, S. S. Miller, P. T. Mocanu, and M. O. Reade, "On a Briot-Bouquet differential subordination," in General Inequalities 3, vol. 64 of Internationale Schriftenreihe Numerische Mathematik, pp. 339-348, Birkhäuser, Basel, Switzerland, 1983.

[11] D. R. Wilken and J. Feng, "A remark on convex and starlike functions," Journal of the London Mathematical Society. Second Series, vol. 21, no. 2, pp. 287-290, 1980.

[12] S. S. Miller and P. T. Mocanu, Differential Subordinations: Theory and Application, vol. 225 of Monographs and Textbooks in Pure and Applied Mathematics, Marcel Dekker, New York, NY, USA, 2000.

[13] J. Patel, "Inclusion relations and convolution properties of certain subclasses of analytic functions defined by generalized Sălăgean operator," Bulletin of the Belgian Mathematical Society. Simon Stevin, vol. 15 , no. 1, pp. 33-47, 2008.

[14] J. Patel, N. E. Cho, and H. M. Srivastava, "Certain subclasses of multivalent functions associated with a family of linear operators," Mathematical and Computer Modelling, vol. 43, no. 3-4, pp. 320-338, 2006.

[15] T. H. MacGregor, "A subordination for convex functions of order $\alpha$," Journal of the London Mathematical Society, vol. 9, no. 4, pp. 530-536, 1975. 


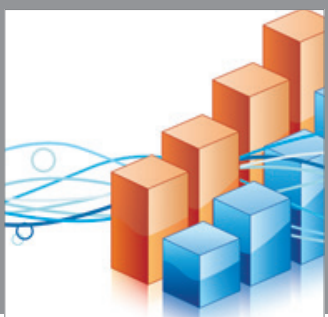

Advances in

Operations Research

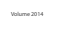

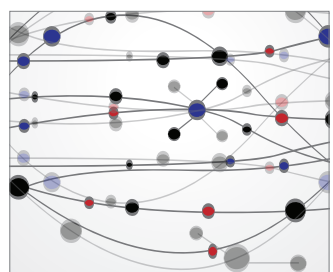

\section{The Scientific} World Journal
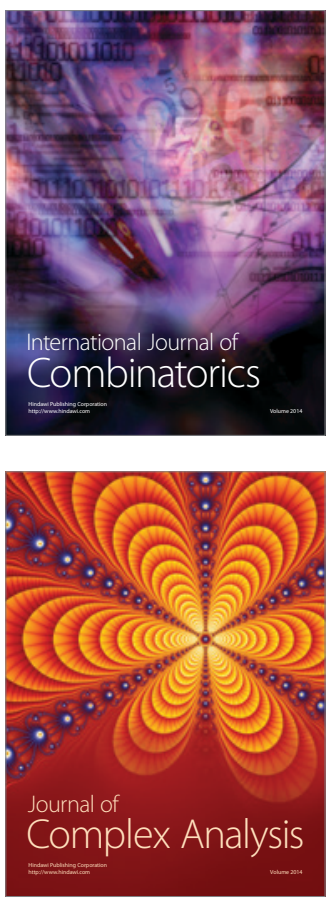

International Journal of

Mathematics and

Mathematical

Sciences
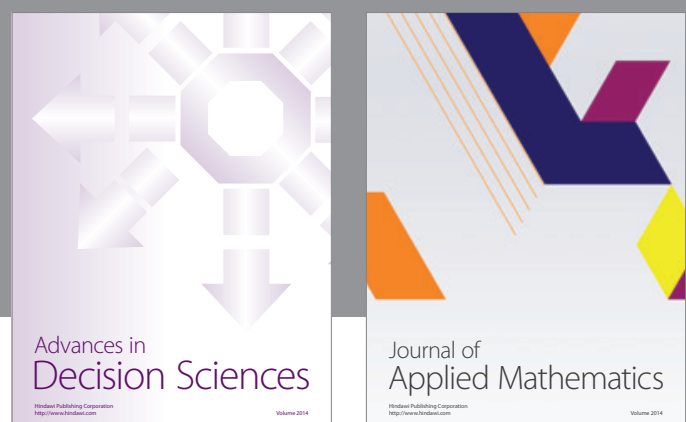

Journal of

Applied Mathematics
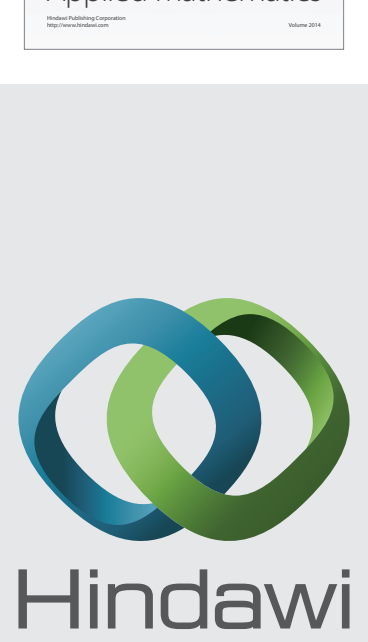

Submit your manuscripts at http://www.hindawi.com
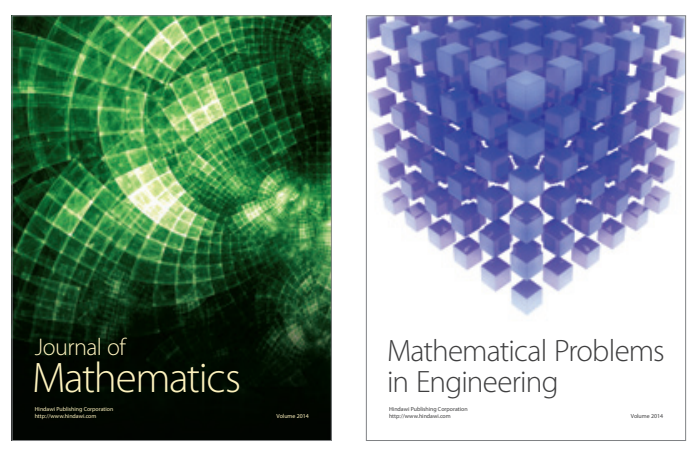

Mathematical Problems in Engineering
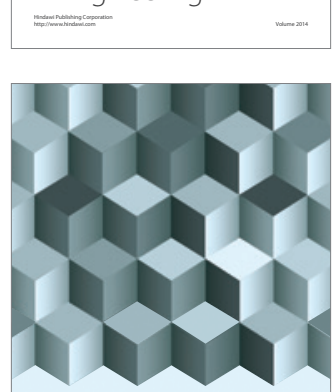

Journal of

Function Spaces
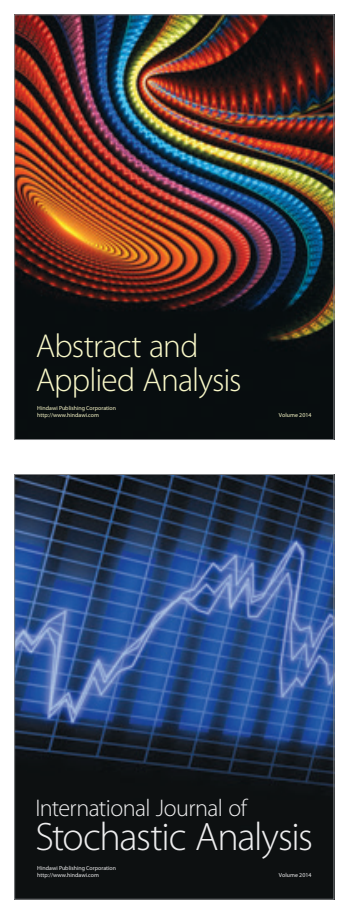

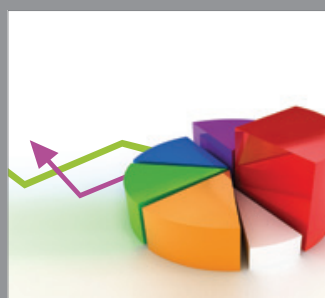

ournal of

Probability and Statistics

Promensencen
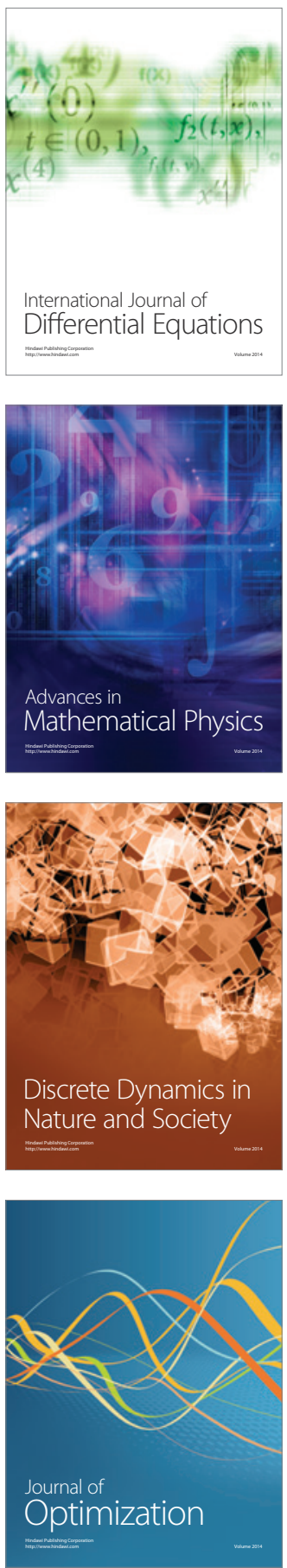